\title{
Médiévales
}

Langues, Textes, Histoire

65 | automne 2013

Le couple dans le monde franc

\section{Le couple dans les stratégies compétitives de la Francie occidentale du XI ${ }^{\mathrm{e}}$ siècle}

Couple and Competitive Strategies in West Francia during the Eleventh Century

\section{Emmanuelle Santinelli-Foltz}

\section{(2) OpenEdition}

Journals

Édition électronique

URL : https://journals.openedition.org/medievales/7106

DOI : 10.4000/medievales.7106

ISSN : 1777-5892

Éditeur

Presses universitaires de Vincennes

Édition imprimée

Date de publication : 1 décembre 2013

Pagination : 77-92

ISBN : 978-2-84292-396-9

ISSN : 0751-2708

Référence électronique

Emmanuelle Santinelli-Foltz, « Le couple dans les stratégies compétitives de la Francie occidentale du xle siècle », Médiévales [En ligne], 65 | automne 2013, mis en ligne le 20 janvier 2014, consulté le 22 avril 2022. URL : http://journals.openedition.org/medievales/7106 ; DOI : https://doi.org/10.4000/

medievales.7106 
Médiévales 65, automne 2013,p. 77-92

Emmanuelle SANTINELLI-FOLTZ

\section{LE COUPLE DANS LES STRATÉGIES COMPÉTITIVES DE LA FRANCIE OCCIDENTALE DU XI ${ }^{\mathrm{e}}$ SIÈCLE}

Après avoir envisagé la compétition qui se jouait autour du lien matrimonial dans la Francie occidentale du $\mathrm{XI}^{\mathrm{e}}$ siècle $^{1}$, ce numéro de Médiévales me donne l'occasion de poursuivre l'étude en envisageant la place du couple dans les stratégies compétitives. Le mariage n'est, en effet, souvent qu'une étape certes importante - de la compétition qui oppose les élites et se poursuit ensuite : les liens matrimoniaux créés peuvent même, parfois, la favoriser. Or, le mariage modifie quelque peu la place et le rôle des individus: si les époux continuent d'appartenir à leur famille respective, ils forment aussi une nouvelle unité de vie, avec ses intérêts et ses ambitions propres. Il s'agit donc ici d'analyser la manière dont le couple se positionne dans ces enjeux : à une époque marquée par l'association plus étroite des épouses à l'exercice du pouvoir de leur mari ${ }^{2}$, l'intervention du couple dans les rivalités pour le pouvoir et les richesses se faitelle de manière solidaire? Comment les deux membres du couple interviennentils dans ce cadre ? Liés à deux familles qui peuvent être en compétition, comment

1. E. Santinelli, «Mariage, compétition et genre dans la Francie occidentale du $\mathrm{XI}^{\mathrm{e}}$ siècle», dans S. Joye éd., Compétition et genre, Turnhout, 2013 (à paraître). La Francie occidentale du $\mathrm{XI}^{\mathrm{e}}$ siècle correspond en effet à un moment où, d'une part, l'Église cherche à encadrer plus étroitement le mariage, ce qui la conduit à s'intéresser davantage aux conjoints et, d'autre part, les femmes, participant plus étroitement à l'exercice du pouvoir royal et aristocratique, apparaissent plus fréquemment aux côtés des hommes dans la documentation.

2. Voir notamment R. LE JAN, Famille et pouvoir dans le monde franc ( $\mathrm{VII}^{e}-\mathrm{X}^{e}$ siècle). Essai d'anthropologie sociale, Paris, 1995, p. 356-366; EAD., «L'épouse du comte du $\mathrm{IX}^{\mathrm{e}}$ au $\mathrm{XI}^{\mathrm{e}}$ siècle: transformation d'un modèle et idéologie du pouvoir», 1999, rééd. dans EAD., Femmes, pouvoir et société dans le haut Moyen Âge, Paris, 2001, p. 21-29; T. Evergates éd., Aristocratic Women in Medieval France, Philadelphie, 1999. 
se positionnent-ils alors dans ce cas ? Cela revient donc à s'interroger sur les relations au sein du couple, mais aussi entre celui-ci et les groupes familiaux auxquels il est lié. Les informations sont cependant déformées par le prisme $\mathrm{du}$ regard et des intentions des auteurs, religieux pour la plupart donc avec une vision - voire pour certains une expérience - particulière du couple et de la vie en couple: analyser leurs discours et leurs silences revient donc aussi à s'interroger sur la représentation qu'ils s'en font.

Dans cette étude, j'envisagerai successivement trois cas de figure: d'abord celui où le couple apparaît solidaire; ensuite celui où les conjoints, tout en étant liés, se trouvent investis différemment dans la compétition; enfin, celui où ils sont en compétition l'un contre l'autre.

\section{Le couple solidaire dans la compétition : ensemble, mais en respectant la hiérarchie entre les sexes?}

Si la documentation pointe d'abord l'objectif sur les hommes, elle présente régulièrement le couple agissant conjointement: le mariage qui octroie à la femme une nouvelle place aux côtés de son époux la conduit à participer avec lui aux compétitions dans lesquelles celui-ci s'inscrit, ce qui n'exclut pas qu'elle contribue à leurs choix. Dans ce contexte, le rôle de l'un et de l'autre peut être plus ou moins actif. Les quelques exemples suivants en témoignent.

\section{Ensemble pour définir les stratégies familiales}

La documentation montre parfois des époux choisissant ensemble pour leur enfant, parmi les prétendant-e-s possibles, celui ou celle qui leur semble être le meilleur parti, ce qui relève des stratégies familiales, compétitives par définition. Ainsi en est-il des parents de Godelieve lorsqu'ils décident vers 1067 de marier leur fille, si l'on suit la Vie de la sainte, rédigée vers 1084 par Drogon, moine de Saint-Winock de Bergues, contemporain des faits ${ }^{3}$ :

Parmi ceux qui demandaient la main de cette vierge si exceptionnelle, il y en avait un du nom de Bertholf [...] dont la dot plus que celle des autres prétendants plut aux deux (utri) parents [de Godelieve]: la jeune fille lui fut promise en mariage légitime ${ }^{4}$.

3. M. CoEns, «La vie ancienne de sainte Godelive de Ghistelles par Drogon de Bergues», Analecta Bollandiana, 44 (1926), p. 102-137 (p. 112 et 114); B. L. VenARde, «Drogo of SintWinoksbergen, Life of St Godelieve», dans T. HEAD éd., Medieval hagiography. An Anthology, New York, 2001, p. 359-373 (p. 359).

4. Vita sanctae Godeliph (BHL 3592), c. 2 (éd. M. Coens, «La vie ancienne de sainte Godelive...», p. 125-137 [p. 127-128]; trad. angl. B. L. VenARdE, «Drogo...», p. 363-373 [p. 364]). 
L'auteur, qui s'intéresse d'abord à Godelieve - mise en avant par les nombreux prétendants qui souhaitent obtenir sa main -, n'en attribue pas moins le choix de l'élu aux deux parents (utri parentes) de la sainte qui se prononcent en faveur de celui qui leur paraît pouvoir mieux contribuer à leurs ambitions, alors que la plupart des sources n'évoquent que le rôle du père (ce qui n'exclut pas une participation de la mère, même si c'est alors en retrait). Le rôle du mariage dans les stratégies familiales et celui de la parenté maternelle dans le système d'alliance laissent néanmoins supposer que l'association de la mère devait être fréquente, ce que la vie de Godelieve précise explicitement.

\section{Ensemble pour asseoir le pouvoir en fondant des monastères}

La documentation montre aussi régulièrement les couples fondant solidairement des communautés monastiques, dont la recherche récente a montré qu'elles constituaient autant des points d'appui politiques destinés à légitimer l'autorité des fondateurs et à ancrer territorialement leur pouvoir que des lieux spirituels, ce qui les intègre dans des stratégies compétitives. Ainsi en est-il de la fondation, en 1047, de l'abbaye féminine de Saintes par le comte d'Anjou, Geoffroi Martel, et son épouse Agnès. Si la charte, reproduite dans le cartulaire de l'abbaye, met en avant Geoffroi Martel, principal détenteur de l'autorité, en utilisant la formulation classique: «Moi, Geoffroi, comte, et mon épouse Agnès ${ }^{5} »$, elle met ensuite tous les verbes au pluriel (deliberavimus, confirmamus, ajunximus, etc.) - sauf lorsqu'il s'agit de donations personnelles de l'un ou de l'autre - et place le signum d'Agnès à la suite de celui de Geoffroi. En outre, tous les privilèges épiscopaux et pontificaux postérieurs font de la fondation celle du comte d'Anjou Geoffroi et de son épouse, la comtesse Agnès ${ }^{6}$, indissociablement liés. La fondation est manifestement perçue comme celle du couple. Il faut dire que les deux époux se trouvent associés à la Saintonge par des biais différents : Geoffroi Martel tient la région en fief de Guillaume VII d'Aquitaine, fils d'Agnès, né de son premier mariage avec le duc Guillaume V (1030) ${ }^{7}$. Les deux époux comme la future abbaye ont tout intérêt à placer la fondation sous ce double patronage: l'abbaye se trouve ainsi étroitement liée à l'un comme à l'autre des époux; quant au couple princier, il entend bénéficier en retour d'un soutien de la communauté, sans compter le prestige procuré par un tel acte. La fondation témoigne donc, sur ce plan, de l'association des deux époux, solidaires dans le climat compétitif ambiant qui oppose les élites princières, non seulement

5. Cartularium Sancte Marie Sanctonensis, $\mathrm{n}^{\circ} 1$ (T. Grasilier éd., Cartulaires inédits de Saintonge, t. II, Cartulaire de l'abbaye royale de Notre-Dame de Saintes, Niort, 1871, p. 1-5).

6. Ibid., n 2-5 (1047-1095), p. 6-11.

7. L. Halphen, Le Comté d'Anjou au XI siècle, Paris, 1906, p. 56-61; O. Gulllot, Le Comte d'Anjou et son entourage au XI' siècle, t. 1, Paris, 1972, p. 39 et 52-53. 
entre elles, mais aussi à l'aristocratie d'un rang moindre, prête à contester leurs prétentions.

S'il est difficile ici de savoir exactement comment la décision a été prise et quel a été le degré d'implication de chacun des époux, il arrive que les sources soient plus précises, à l'image de la Chronique de Maillezais, rédigée par un moine de l'abbaye entre 1067 et 1072-1073. Celle-ci montre le couple fondateur discuter ensemble, le duc Guillaume IV interroger sa femme Emma sur les conseils qu'elle pourrait lui donner, et celle-ci, «devenue sa propre chair», lui suggérer ce qu'elle pense devoir être fait (s'il le commande), ce qui lui convient pleinement: à elle la restauration du monastère de Maillezais et le soin des âmes; à lui les tâches de la guerre et la construction d'un fort ${ }^{8}$. L'auteur, qui replace comme souvent le récit de la fondation dans un contexte miraculeux (dont a été témoin Emma qui en a informé son mari), doit cependant, pour paraître plausible, l'inscrire dans des cadres réels: ici, celui de la décision d'un couple, envisagé comme union charnelle (ce que souligne la référence à l'Évangile de saint Matthieu, 19,5), mais aussi comme association, dans laquelle la femme apparaît comme un partenaire à part entière. Cette association n'exclut, cependant, ni le partage des tâches (la défense militaire à Guillaume/le salut des âmes à Emma), ni la hiérarchie à respecter au sein du couple (Emma conseille/ son mari commande), ce qui est conforme à la conception que l'on avait alors des relations entre les sexes ${ }^{9}$.

\section{Ensemble pour éliminer les couples rivaux}

Si le rôle des fondations monastiques dans les stratégies compétitives est le plus souvent implicite, notamment lorsqu'elles sont effectuées par des couples, la documentation met parfois explicitement en lumière l'association des époux dans le cadre de véritables luttes de pouvoir. Ainsi, Adémar de Chabannes précise, dans sa Chronique (composée entre 1024-1025 et 1029), que, dans la compétition qui oppose les comtes d'Anjou et du Maine:

Foulques, comte d'Anjou, incapable de l'emporter ouvertement sur Herbert, comte du Mans, fils d'Hugues, l'attira par ruse (dolo) avec lui [...]. Le même jour, l'épouse de Foulques tenta de s'emparer par ruse (dolo) de la femme d'Herbert, avant que la nouvelle de la trahison dont Herbert avait été victime ne lui fût parvenue ${ }^{10}$.

8. Qualiter fuit constructum Malleacense et corpus sancti Rigomeri translatum, I [A] (éd. et trad. G. Pon, Y. Chauvin, La Fondation de l'abbaye de Maillezais. Récit du moine Pierre, La Rochesur-Yon, 2001, p. 102-103). Pour la datation, p. 16-17.

9. Entre autres, R. LE JAN, «L'épouse du comte...».

10. Adémar de Chabannes, Chronicon, III, 64 (éd. P. Bourgain, Turnhout, 1999, p. 184 ; trad. Y. Chauvin et G. Pon, Chronique, Turnhout, 2003, p. 284). 
Même si l'auteur, hostile à Foulques, présente les choses à sa manière ${ }^{11}$, il établit, pour ces événements de 1025 dont il est contemporain, un parallèle entre le comte d'Anjou et son épouse, associés pour s'emparer du couple comtal du Maine et usant de méthodes similaires (la ruse). En précisant que Foulques et son épouse cherchent à s'emparer de la femme d'Herbert, après avoir capturé celui-ci, il peut laisser supposer que celle-ci est susceptible de poursuivre seule la compétition avec l'Anjou, ou du moins de constituer un obstacle aux ambitions du comte $\mathrm{d}^{\prime}$ Anjou ${ }^{12}$. D'autres sources confirment en effet, pour le $\mathrm{XI}^{\mathrm{e}}$ siècle, que l'épouse se substitue à son mari lorsque celui-ci n'est plus en mesure d'exercer l'autorité ${ }^{13}$. Si l'auteur met en avant l'action conjointe du couple comtal, il ne place cependant pas les comtes et comtesses sur le même plan. D'une part, les comtesses ne sont pas nommées, ce qui revient à les considérer comme les exécutantes de projets décidés par leur mari, donc comme des collaboratrices plutôt que comme de véritables associées. D'autre part, Adémar présente la compétition comme étant d'abord une rivalité opposant les deux comtes: l'épouse de Foulques Nerra (Hildegarde) n'intervient que dans un second temps (une fois Herbert maîtrisé par Foulques); l'épouse d'Herbert (nommée Hildegarde aussi) n'entre en jeu que lorsque son mari n'est plus en mesure de participer à la compétition et qu'elle se substitue alors à lui. Si les liens matrimoniaux conduisent les épouses à participer activement aux enjeux compétitifs aux côtés de leurs époux, les sources n'en font souvent que des auxiliaires, conformément à la hiérarchie qui existe entre les sexes au sein du couple. Reste à savoir si cela correspond à la réalité ou si cela ne relève que du discours des auteurs, tous des hommes issus du même milieu.

\section{Ensemble face aux risques de la compétition}

L'extrait précédent suggère en outre que l'épouse partage avec son mari les risques de la compétition: d'autres sources le mettent explicitement en lumière, ce qui tend à pencher en faveur d'une association étroite de l'épouse aux enjeux compétitifs. Selon la Chronique Sénonaise (compilation réalisée vraisemblablement au début du XII ${ }^{\mathrm{e}}$ siècle ${ }^{14}$ ), après la prise du château de Melun

11. B. Bachrach, Fulk Nerra, the Neo Roman Consul, 987-1040. A Political Biography of the Angevin Count, Londres, 1993, p. 173-177.

12. B. BACHRACH a néanmoins montré qu'elle n'était pas en capacité de le faire, ibid., p. 173 174.

13. Entre autres T. Evergates éd., Aristocratic Women..., et E. Santinelli, Des femmes éplorées? Les veuves dans la société aristocratique du haut Moyen Âge, Lille, 2003, p. 373-374.

14. R. H. Bautier, M. Gilles, Chronique de Saint-Pierre de Sens, dite de Clarius, Paris, 1979, p. VII et IX-XI. 
par le roi Robert, en 999, «Gautier et sa femme [qui avaient pris parti pour Eudes II contre Robert et lui avaient livré le château] furent pendus au gibet $\gg^{15}$.

La compétition comporte en effet des risques: il y a souvent un vainqueur et un vaincu. Il arrive donc aussi à l'épouse d'être victime des compétitions dans lesquelles son mari, avec ou sans elle, se lance. Davantage associée aux compétitions, l'épouse en partage régulièrement les conséquences en cas de défaite: ici, la pendaison pour l'un comme pour l'autre, pour avoir trahi Robert et être passés - par des actes réalisés de concert (ils ont pris parti ensemble, puis livré le château) - au parti rival. Si le couple peut subir le même sort, l'épouse peut être victime de sanctions qui lui sont propres, même si celles-ci visent d'abord à atteindre le mari. La Chronique de Maillezais évoque ainsi, vers 1070, la compétition qui, à l'extrême fin du $x^{\mathrm{e}}$ siècle (v. 997), oppose Guillaume V, duc d'Aquitaine, et Boson, comte de la Marche: Boson attaque d'abord un château $\mathrm{du}$ duc qui riposte. Boson s'enfuit alors et Guillaume $\mathrm{V}$ attaque à son tour un château de Boson qui est pris d'assaut:

On y découvrit l'épouse de Boson, que le duc remit à la garde de ses fidèles. Il s'opposa sévèrement à la débauche des jeunes qui formaient la garde princière et voulaient, pour faire affront au mari, qu'on la livrât à leurs outrages (I, H, p. 121).

Même si l'auteur commet des confusions ${ }^{16}$ et qu'il cherche surtout à montrer la grandeur de Guillaume V, il n'en présente pas moins, dans un contexte de compétition qui oppose d'abord les hommes, le viol de l'épouse comme une stratégie de guerre et de gouvernement: peu mentionné dans les sources, ce qui ne signifie pas qu'il ait été rare, le viol apparaît comme une menace destinée à freiner la participation du mari aux compétitions. Il n'empêche que, même si l'objectif est de punir les ambitions excessives du mari, c'est sa femme qui le subit, parce que femme et épouse ${ }^{17}$.

Si le couple apparaît régulièrement de manière solidaire dans les enjeux compétitifs - sans pour autant forcément y prendre part de la même façon -, il lui arrive aussi d'y intervenir de manière très différente.

15. Chronicon Sancti Petri Vivi (éd. et trad. R. H. Bautier et M. Gilles, Chronique de SaintPierre de Sens..., p. 105).

16. Voir G. Pon, Y. Chauvin, La Fondation..., p. 21-22: il s'agit en fait d'Aldebert de la Marche (et non de Boson).

17. R. Branche, F. Virgili dir., Viols en temps de guerre, Paris, 2011, notamment p. 10-11, même si les analyses concernent d'abord l'époque contemporaine. 


\section{Époux et épouse investis différemment dans la compétition : réalité ou discours?}

Les sources mettent en lumière certaines compétitions dans lesquelles époux et épouse, mentionnés l'un et l'autre, jouent un rôle différent, ce qui ne signifie pas qu'ils ne sont pas impliqués dans le même processus ni qu'ils n'agissent pas dans le même intérêt. Deux cas de figure ont été observés.

\section{L'épouse, instrument revendicatif dans les compétitions engagées par son mari}

La documentation montre que, dans certains cas, l'épouse apparaît comme un instrument utilisé par son mari dans la compétition, sans que l'on perçoive toujours le rôle précis qu'elle y joue. Ainsi, selon Raoul Glaber, qui écrit ses Histoires dans les années 1030-1040:

Leur prince [aux Bretons] était autrefois un certain Conan qui épousa la sœur de Foulques, comte des Angevins, et devint encore plus odieux que les autres princes de cette nation. S'étant fait couronner à la manière d'un roi, il infligea aux pauvres gens de cette région perdue les tourments divers d'une tyrannie stupide [il est ensuite en conflit avec Foulques Nerra, ce qui se solde par sa défaite à Conquereuil (1016) et sa mort] ${ }^{18}$.

L'auteur semble établir un lien entre le mariage (vers 970) de Conan I (duc des Bretons, v. 970-992) avec la sœur de Foulques Nerra (comte d'Anjou, 9871040), non nommée (Ermengarde) ${ }^{19}$, et le fait qu'il devient plus odieux que les autres princes bretons: comme si le fait d'avoir épousé cette femme permettait à Conan de se considérer comme supérieur aux autres, jusqu'à leur imposer son autorité et même rivaliser avec son beau-frère. Si l'auteur propose de voir dans le mariage avec Ermengarde une explication des velléités de Conan, il ne précise pas si Ermengarde a poussé son mari dans ce sens ou si celui-ci s'est seulement servi du surcroît de prestige qu'elle lui a apporté. Dans le même ordre d'idées, les sources évoquent parfois - même si ces cas sont minoritaires - des maris qui entrent en compétition, militairement ou judiciairement, de manière réelle ou feinte, avec des groupes familiaux ou des communautés religieuses liés à leur épouse, au nom des «droits », prétendus ou réels, de celle-ci. S'agit-il cependant de décisions et d'actions du seul mari qui utilise tous les leviers à sa disposition sans y associer sa femme, ou du discours filtré des sources qui n'éclaire que le mari, alors que les décisions et actes sont ceux d'un couple?

18. Raoul Glaber, Historiae, 1. II, III, 4 (éd. et trad. M. Arnoux, Histoires, Turnhout, 1996, p. 98-99).

19. Voir B. Bachrach, Fulk Nerra ..., p. 11, 14-15 et 37-46. 
En effet, si la documentation réduit généralement les épouses, présentées comme «instrument revendicatif», à un rôle passif, elle mentionne aussi parfois l'influence directe de l'épouse, ce qui invite à nuancer leur passivité apparente. Si l'on en croit Dudon de Saint-Quentin, clerc vermandois (v. 960-965-av. 1043) proche des ducs de Normandie, l'initiative du conflit qui oppose, entre 961 et 965, le comte de Blois, Thibaud le Tricheur, au comte de Rouen, Richard I ${ }^{\text {er }}$, reviendrait au premier qui y aurait été poussé par la furie de la belle-mère (furiis novercalibus) du second ${ }^{20}$, c'est-à-dire Liégearde (à la fois ex-bellemère de Richard, dont elle avait épousé le père en premières noces, et épouse en deuxièmes noces de Thibaud le Tricheur) ${ }^{21}$. L'auteur, qui écrit à la demande de Richard ${ }^{22}$, reporte la responsabilité du conflit sur l'autre camp - ce qui n'est pas très surprenant - et plus particulièrement sur Liégearde, inspiratrice de cette nouvelle épreuve compétitive: la situation se trouve en quelque sorte inversée, le mari se trouvant transformé en instrument de la colère de sa femme. Le parti pris de Liégearde peut s'expliquer par les droits qu'elle a conservés en Normandie de son premier mariage, qui étaient peut-être menacés. En revanche, il est peu probable qu'il s'agisse là des seules raisons du conflit, à lier de manière plus générale à la brutale affirmation de la maison de Blois aux dépens de ses voisins. Il n'en demeure pas moins que Dudon fournit des explications qui doivent être plausibles pour ses contemporains du début $\mathrm{du} \mathrm{XI}^{\mathrm{e}}$ siècle - dont certains ont d'ailleurs été témoins des faits - et qui mettent en avant l'influence de l'épouse dans les décisions de son mari. Néanmoins, si la responsabilité de la décision se trouve ainsi reportée sur celle-ci, ce n'est pas elle que l'auteur montre ensuite décider et agir: la hiérarchie reconnue par tous au sein du couple place la femme, conseillère et soutien, sous l'autorité du mari, décideur et principal acteur. Il n'en reste pas moins que les revendications d'un homme au nom des droits de sa femme cachent probablement des décisions de couple, dont les enjeux dépassent généralement les seules questions de droits, de propriété ou de revenus, pour relever autant du pouvoir que de l'honneur.

20. Dudon de Saint-Quentin, De moribus et actis primorum Normaniae ducum (éd. J. LaIR, Mémoires de la Société des Antiquaires de Normandie, 23, [1865], 103, p. 265 ; trad. angl. E. Christiansen, History of the Normans, Woodbridge, 1998, IV, 75, p. 139). Sur ce conflit, voir entre autres P. Baudouin, La Première Normandie ( $X^{e}-X I^{e}$ siècles), Caen, 2004, p. 166-170; F. Neveux, La Normandie des ducs aux rois, $X^{e}$-XII ${ }^{e}$ siècle, Rennes, 1998, p. 48-51.

21. Sur Liégearde, voir E. SANTineLLI, «Liégearde (v. 983-984), fille, épouse, veuve et mère de princes, dans la Francie occidentale du $\mathrm{x}^{\mathrm{e}}$ siècle», dans Femmes d'exception au Moyen Âge, Actes du colloque de Saint-Guilhem-le-Désert (26 mai 2012), à paraitre.

22. Entre 996 et 1015, puis complété après 1015, à la demande des fils de Richard, P. BAudouin, La Première Normandie..., p. 63. 


\section{L'épouse, arbitre dans les compétitions qui impliquent son mari}

Si les sources éclairent d'abord les maris, parce qu'en tant qu'hommes il leur revient de réaliser les gestes publics qui engagent, il arrive néanmoins que la documentation mette en avant le rôle de l'épouse, promue au rang d'arbitre de la compétition opposant son mari à un rival. En effet, si les décisions, notamment pour départager des concurrents, sont le plus souvent présentées comme celles d'un homme, parfois - est-il précisé - prises avec le conseil de son entourage et notamment de son épouse, certaines se trouvent justifiées par les auteurs qui les attribuent à des femmes et montrent les hommes s'y rallier. Ainsi en est-il de la succession du roi Robert I ${ }^{\text {er }}$, rapportée comme suit par Raoul Glaber dans ses Histoires:

[Hugues le Grand] voyant le royaume sans roi et sachant que l'établissement d'un roi dépendait de sa volonté, fit demander à sa sœur [Emma, épouse de Raoul, fils du duc Richard de Bourgogne] lequel de son frère ou de son mari, Raoul, devait, selon elle, recevoir le trône. Sagement, elle répondit qu'elle préférait embrasser le genou de son mari plutôt que de son frère. Hugues, reconnaissant, donna son assentiment à ce choix et laissa Raoul s'établir sur le trône (1. I, II, 6, p. 50-51).

L'événement remonte à 923 et l'explication de l'élection à la royauté de Raoul est propre à l'auteur. Il n'en montre pas moins, à ses lecteurs du XI ${ }^{\mathrm{e}}$ siècle, deux hommes en compétition pour le trône et l'arbitrage, sage, d'une femme qui privilégie son mari (et les siens) aux dépens de son frère (et donc de ses consanguins). Même s'il s'agit pour l'auteur de reporter la responsabilité de ce choix sur une femme (dont le nom n'est même pas précisé), de manière à taire les vraies raisons qui ont fait renoncer Hugues à la royauté ${ }^{23}$, l'explication donnée témoigne du rôle de conseil que jouent les femmes au sein du couple et plus largement dans le groupe familial, ainsi que de leur participation active aux décisions relatives aux compétitions dans lesquelles les uns et les autres se trouvent engagés. Elle apparaît aussi comme une invitation faite aux épouses à soutenir et à privilégier leur mari avant leur parenté consanguine: la femme mariée quitte sa famille pour s'établir chez son mari - la résidence virilocale constituant la norme - et, si les liens avec sa famille ne sont pas rompus, elle fait désormais partie d'une unité familiale qui la lie davantage à son mari et à la famille de celui-ci qu'à la sienne. Cela se traduit aussi dans la manière dont l'épouse se positionne s'il y a compétition entre l'une et l'autre, alors que la question de ce positionnement ne se pose pas pour l'époux. La priorité à accorder par l'épouse à son mari, suggérée par Raoul Glaber, n'est nullement étonnante sous la plume d'un moine, à une époque où l'Église s'attache à promouvoir le couple conjugal

23. Y. SASSIER, Hugues Capet, Paris, 1987, p. 89-90. 
et à limiter la pression des groupes familiaux ${ }^{24}$. Il n'en demeure pas moins qu'il en résulte, du moins dans le discours, des conséquences différentes pour les deux époux: les enjeux compétitifs ne se posent pas de la même manière pour chacun des partenaires du couple.

Malgré l'image reflétée par la documentation de couples solidaires dans les conflits qui les opposent à des compétiteurs, avec une insistance particulière sur le soutien attendu de l'épouse à son mari, il arrive aussi qu'elle évoque des couples qui s'illustrent par leur compétition interne.

\section{Époux et épouse en compétition : compétition conjugale ou familiale?}

Si le lien matrimonial conduit le plus souvent mari et femme à se retrouver côte à côte, il arrive que certaines compétitions les voient s'affronter, sans que l'on perçoive toujours clairement les enjeux qui se cachent derrière ces affrontements. Ces compétitions, aux formes diverses ${ }^{25}$, ont des issues plus ou moins dramatiques.

\section{Compétition à mort}

La documentation offre quelques exemples de couples où l'un des époux en vient à assassiner l'autre. Si l'on en croit la Vie de Godelieve, Bertholf, qui vient d'être fiancé à Godelieve, commença à la haïr avant même de l'avoir ramenée chez lui (c. 2). Si sa mère le pousse ensuite à poursuivre dans cette voie (c. 3), c'est lui - soutenu par des parents et des partisans - qui ordonne de la maltraiter (c. 4-5), ce qui provoque - malgré la compassion des femmes du voisinage et des épouses de ses parents par alliance (affinitate coniunctae) - la fuite de Godelieve qui revient chez son père; et c'est lui qui, après avoir été contraint par le comte et l'évêque de reprendre son épouse (c. 6), sans pour autant mieux la traiter (c. 7-8), finit ensuite par élaborer un plan pour la faire tuer, sans en être accusé, seul moyen pour lui de s'en débarrasser (c. 9-12). La Vita et ce qu'elle dit du mariage au Xi ${ }^{\mathrm{e}}$ siècle ont été analysés par Georges Duby ${ }^{26}$ : je ne retiendrai donc que ce que ce texte évoque quant aux rivalités entre époux, qui prennent ici la forme d'une compétition à mort. Si l'auteur de la Vita focalise son attention sur eux, opposés de façon manichéenne (la pieuse Godelieve/la brute

24. R. LE JAN, Famille..., notamment p. 280 et 315.

25. Sont ici exclues les compétitions qui mettent en lice un ou une rivale, analysées dans E. SANTinelli, «Mariage, compétition et genre...».

26. G. Duby, Le Chevalier, la femme, le prêtre. Le mariage dans la France féodale, 1981, rééd. ID., Féodalités, Paris, 1996, p. 1262-1263. 
criminelle Bertholf), et s'il soulève la question de l'incompatibilité d'humeur entre des époux qui ne se sont pas choisis - même si Bertholf a défendu sa candidature pour obtenir Godelieve -, il témoigne aussi que ceux-ci se trouvent insérés dans des groupes qui les soutiennent - voire les poussent - plus ou moins activement, groupes formés de parents, amis, fidèles, domestiques, voisins, religieux, etc. Même s'il est difficile de connaître les enjeux véritables de cette histoire, celle-ci se trouve très certainement liée aux rivalités qui opposent le comte de Boulogne (probablement seigneur du père de Godelieve) au comte de Flandre (seigneur de Bertholf), qui cherche à mieux contrôler la Flandre maritime, notamment en poussant ses vassaux à se lier par mariage aux familles locales ${ }^{27}$. Si la compétition prend ici un tour dramatique, ce qui semble assez exceptionnel au vu de la documentation (que la victime soit l'épouse ou - plus rarement - l'époux), le fait qu'elle soit rarement circonscrite au seul couple est confirmé par d'autres «affaires». Deux dossiers, aux conséquences moins tragiques, ressortent du corpus et montrent en outre que les stratégies peuvent être différemment déployées par le mari et la femme.

\section{Les démêlés de Guillaume IV et Emma}

Le premier de ces conflits internes au couple met en rivalité Guillaume IV d'Aquitaine et Emma, dont les démêlés conjugaux (entre 977 et 996) - confirmés par les actes diplomatiques - sont rapportés un siècle plus tard par la chronique de Maillezais (dans la mesure où ils ont des répercussions sur les débuts du monastère). Élisabeth Carpentier a déjà fait le point sur l'histoire de ce «couple tumultueux ${ }^{28} \gg:$ je ne retiendrai donc ici que ce qui concerne les rapports du couple à la compétition, en partant du récit du moine Pierre, reflet des conceptions de la seconde moitié du XI ${ }^{\mathrm{e}}$ siècle. C'est une rumeur d'adultère du duc avec la femme du vicomte de Thouars qui serait «l'aliment de la haine [qui s'insinua] dans le cœur des deux époux» : c'est du moins l'excuse que l'auteur avance, compréhensible pour ses contemporains, ce qui témoigne des difficultés de la vie conjugale au quotidien, à une époque où le mariage - qui relève des stratégies familiales - n'aboutit pas forcément à une union heureuse, ou du moins suffisante sur tous les plans.

Dès l'instant où la comtesse eut appris la honte de ce scandale, aussitôt elle commença à se montrer désagréable pour son mari et à lui reprocher journellement

27. M. Coens, «La vie ancienne... », p. 119-122; G. Duby, Le Chevalier, la femme ..., p. 12601261.

28. É. CARPENTIER, «Un couple tumultueux en Poitou à la fin du $x^{\mathrm{e}}$ siècle: Guillaume de Poitiers et Emma de Blois», dans M. Rouche éd., Mariage et sexualité au Moyen Âge, accord ou crise?, Paris, 2000, p. 203-215. 
le mépris qu'il lui avait témoigné. Celui-ci, impatient de se disculper, multipliait les discours mais quand il se fut rendu compte qu'il ne pouvait, par ses seules paroles, apaiser la futilité féminine, il décida de s'opposer aux plaintes de son épouse en faisant la sourde oreille. Peu de jours après [...], elle se heurte à celle qui, croyait-elle, avait entraîné son mari dans le stupre. Donc se jetant sur elle [...] elle excite ses compagnons à abuser d'elle tout au long de la nuit [...]. Puis Emma, considérant les conséquences, je veux dire la colère implacable de son mari [...], gagna de nuit avec une petite suite le château de Chinon qui alors lui appartenait (I, B, p. 102-105).

La disproportion de la vengeance d'Emma à l'égard des deux incriminés (elle se montre désagréable vis-à-vis de son mari et lui reproche son mépris/elle fait violer la vicomtesse, acte dont on a vu qu'il vise d'abord le mari), sa fuite à Chinon (dans le comté de Blois sous l'autorité de son frère) et le contexte géopolitique invitent cependant à donner une autre dimension à ce conflit qui n'est pas que conjugal ${ }^{29}$. Il s'inscrit dans la compétition acharnée qui oppose le comte de Blois au comte d'Anjou (allié au vicomte de Thouars, vassal du duc d'Aquitaine), tout comme le viol de la vicomtesse de Thouars : celui-ci réalisé sur l'ordre d'Emma, membre de la famille de Blois le mieux placé (en tant que duchesse d'Aquitaine), prétextant un adultère du duc (réel, supposé ou inventé), vise à porter atteinte au comte d'Anjou autant qu'à son allié. Les compétitions entre groupes familiaux ont des prolongements au sein du couple.

La suite de l'histoire montre que la compétition au sein du couple, à replacer dans celle plus large des élites princières, se poursuit. J'en reproduis quelques extraits, le discours de l'auteur n'étant pas sans intérêt:

[...] le peuple de tout le pays avait fini par prendre en horreur l'interminable colère du duc à l'égard de son épouse et ne cessait de répéter que, tant que la comtesse avait vécu à ses côtés, elle avait enveloppé le monde d'une surabondance de biens qui avait reculé au moment de sa fuite [...]. C'est pourquoi le prince [...] renoue avec son épouse; [...] il confesse qu'il a commis de lourdes fautes. [La construction du monastère de Maillezais est achevée avec le soutien d'Emma.] (I, B, p. 107).

[...] Les deux époux se querellent à nouveau, et l'épouse s'entend menacer de nombreuses peines. [...] À nouveau, elle s'enfuit. Retournant chez les siens, elle fit enlever au prince le fils qu'elle avait eu de lui [...]. Pendant ce temps, l'époux [...], par mépris pour sa femme, chassa les moines venus de Tours [qu'Emma avait fait venir, p. 107] (I, E, p. 113-115).

[Le duc Guillaume] fut atteint d'une grave maladie dont certains attribuaient la cause au scandaleux bannissement de son épouse. [Il fait supplier Emma, qui finit par céder, de venir.] [...] le prince [...] fait un retour complet sur lui-même [...] il remit le pouvoir à la mère et au fils (I, E, p. 115).

29. Cela a déjà été suggéré par G. Pon, Y. Chauvin, La Fondation..., p. 177, n. 174. 
Si l'on ne peut totalement exclure l'influence de la mésentente au sein du couple, les séparations et retrouvailles des époux se font surtout au gré des nécessités d'alliance, plus ou moins fortes, entre le comte de Blois et le duc d'Aquitaine. Mais, plus que la réalité des faits, ce qui importe, c'est la façon dont l'auteur les rapporte. Il montre ainsi que le fils du couple (Guillaume V), mais aussi (et surtout) le monastère de Maillezais, auquel Emma est particulièrement attachée, deviennent des enjeux dans la compétition à laquelle se livrent les époux: Emma emmène son fils, seul héritier de son père, lors de sa seconde fuite; le duc s'en prend à Maillezais dès qu'il est en conflit avec sa femme. Le moine Pierre juge aussi l'attitude des uns et des autres et, par ce biais, donne des leçons plus larges à ses contemporains: il condamne la séparation - que l'initiative vienne d'Emma, la première fois, ou de Guillaume, la seconde -, alors que l'Église cherche à imposer plus fermement l'indissolubilité du mariage ; mais il insiste aussi sur les «lourdes fautes» avouées du duc et explique tous ses malheurs par l'absence d'Emma, avertissement implicite à tous les aristocrates masculins des dangers de l'adultère et de la séparation, pour leur vie comme pour l'exercice de leur autorité. Le couple apparaît ainsi comme une entité à défendre et à protéger contre les dangers susceptibles de le menacer, de l'extérieur (pressions familiales) comme de l'intérieur (adultère).

\section{Robert le Pieux et Constance: affrontement d'époux - chefs de partis ou de champions désignés?}

La seconde «affaire», qui voit s'affronter entre 1025 et 1030 le roi Robert le Pieux et la reine Constance ${ }^{30}$, est évoquée notamment par Raoul Glaber dans ses Histoires. La première épreuve qui oppose les époux porte, après la mort de leur aîné Hugues (1025), sur le choix de l'héritier parmi leurs fils. Robert se prononce en faveur d'Henri, «né après Hugues»:

Mais à nouveau, sa mère, saisie d'une fureur féminine, s'éleva contre son père et contre tous ceux qui l'appuyaient, affirmant que le plus apte à gouverner le royaume était le troisième fils, nommé Robert, du nom de son père. [...] Le roi, rassemblant dans la cathédrale de Reims les grands du royaume, ceignit de la couronne celui qu'il avait choisi, Henri (III, 34, p. 208-209).

Robert et Constance soutiennent donc chacun un fils différent, ce que confirment aussi quelques lettres adressées à Fulbert de Chartres (partisan du roi) ou envoyées

30. A. W. LewIS, Le Sang royal. La famille capétienne et l'État, France, $X^{e}$-XIV siècle, trad. fr., Paris, 1986, p. 51-52; L. TheIs, Robert le Pieux. Le roi de l'an mil, Paris, 1999, p. 232-238; J. Dhond, «Sept femmes et un trio de rois», Contribution à l'histoire économique et sociale, 3 (1964-1965), p. 37-70 (p. 50-51); ID., «Élection et hérédité sous les Carolingiens et les premiers Capétiens», Revue belge de philologie et d'histoire, 18/4 (1939), p. 913-953 (p. 938-948). 
par lui ${ }^{31}$. Dans cette compétition dont les motivations réelles restent floues, chacun dispose de soutiens, notamment dans le milieu princier: il n'y a pas de différence dans les stratégies au sein du couple selon le sexe du compétiteur. Certains princes hésitent néanmoins à prendre parti (craignant les représailles de la partie adverse ${ }^{32}$ ), preuve que la compétition qui oppose le roi et la reine est vive. Ceux qui prennent parti se positionnent en fonction de leurs propres enjeux compétitifs et l'on y retrouve notamment les vieux antagonistes dans des camps opposés (entre autres le comte de Blois, du côté du roi, et celui d'Anjou, du côté de la reine, sa cousine). S'agit-il cependant, comme on le présente généralement, d'une rivalité entre le roi et la reine qui ont chacun constitué un parti? Ou bien de la compétition entre deux groupes qui ont mis en avant deux champions différents, l'un le roi, l'autre la reine, chacun d'eux défendant sa propre marge de manœuvre à la cour en même temps que les intérêts de son groupe? Le roi finit par l'emporter: son point de vue prévaut et c'est donc le fils qu'il a choisi qui est couronné (1027), parce qu'il est roi - et homme -, si l'on comprend bien la remarque de Raoul Glaber, mais aussi parce que son parti était plus puissant que celui derrière la reine. La compétition n'est cependant pas terminée: la seconde épreuve a lieu quelques années plus tard:

Peu de temps après, les deux frères conclurent un pacte d'amitié puis, poussés par l'arrogance de leur mère, se mirent à attaquer les bourgs et les châteaux de leur père $[\ldots]$. Bouleversé et consterné, le roi rassembla une armée $[\ldots]$. Ce fut pire qu'une guerre civile (III, 35, p. 208-209).

Le conflit entre les époux ressurgit en 1030 et se traduit de manière plus violente par leur positionnement dans deux camps différents qui s'affrontent militairement: Constance, à l'instigation de la révolte de ses fils, se trouve de leur côté; Robert, qui se défend et réagit, est contre eux. Les causes de ce conflit intrafamilial, obscures, ne peuvent être réduites au seul caractère odieux et perfide de Constance, «démon femelle» selon J. Dhondt ${ }^{33}$. Même si les relations entre Robert et Constance n'ont jamais été simples et qu'elles deviennent plus conflictuelles à la fin du règne, leurs rivalités relèvent probablement moins de problèmes de couple que d'affrontement entre groupes de parents et d'alliés rivaux dont ils sont des représentants majeurs. Si la documentation évoque la présence de la reine aux côtés de ses fils, ce qui témoigne du soutien qu'elle a pu

31. Fulbert de Chartres, Epistolae, no 122 et 125-126 (éd. et trad. Société archéologique d'Eure-et-Loir, Euvres. Correspondance, controverse, poésie, 2006, p. 29-403 [p. 354-355 et 360363]).

32. Certaines lettres de Fulbert en témoignent: voir la note précédente.

33. J. Dhondt, «Sept femmes...», p. 51; ID., «Une crise du pouvoir capétien, 1032-1034», dans Miscellanea mediaevalia in memoriam Jan Frederik Niermeyer, Groningen, 1967, p. 137-148 (p. 138). 
leur apporter, montrer la reine à l'initiative de la révolte apparaît surtout comme un moyen pour Raoul Glaber de reporter sur une femme la responsabilité des conflits nés de la compétition - désapprouvée - pour le pouvoir entre père et fils. C'est aussi une critique voilée à l'égard de Robert - et des hommes dans la même situation -, incapable de faire respecter la hiérarchie au sein du couple ${ }^{34}$, ce qui conduit à ce type de situation hors norme (sans pour autant être exceptionnelle).

Le corpus témoigne donc que le couple correspond, dans le discours autant que dans la pratique, à une réalité que met notamment en avant la compétition qui oppose les élites au $\mathrm{XI}^{\mathrm{e}}$ siècle. Lorsqu'ils sont évoqués l'un et l'autre, les époux sont ainsi le plus souvent présentés décidant et mettant en œuvre ensemble les stratégies familiales. Cela ne signifie cependant, ni qu'ils interviennent de la même façon (outre qu'il peut y avoir partage des tâches, les principaux actes reviennent généralement à l'époux, secondé par son épouse), ni qu'ils ne soient pas partagés entre la défense de leurs intérêts et ceux de leur groupe familial (lorsqu'il y a rivalité). Cela ne signifie pas non plus que les choses se posent de la même manière pour les deux partenaires du couple: pour l'épouse, cela revient souvent à privilégier son mari et la famille de celui-ci aux dépens de ses consanguins. Si le discours insiste sur un modèle de couple qui privilégie l'association des époux, tout en plaçant l'épouse - soumise - sous l'autorité de l'époux, il témoigne aussi, par les affaires évoquées ou certaines allusions, confirmées parfois par les actes de la pratique, d'une réalité beaucoup plus diversifiée, accordant notamment à l'épouse un rôle beaucoup plus actif, ou mettant en lumière les difficultés de la vie en couple - que les causes en soient internes ou externes -, jusqu'à mettre parfois en compétition les époux entre eux. Enfin, si les stratégies mises en œuvre par les époux dans les compétitions qui les opposent aux autres, mais aussi entre eux, reposent sur les mêmes bases, il arrive qu'elles diffèrent selon le sexe des compétiteurs (utilisation des fils par l'épouse), de même que certaines sanctions (viol de l'épouse).

Emmanuelle Santinelli-Foltz - Université de Valenciennes, Département d'Histoire, Le Mont-Houy, BP 311, 59304 Valenciennes Cedex

34. Raoul Glaber évoque quelques paragraphes avant le fait que Constance dominait son mari (III, 32, p. 202-203). 


\section{Le couple dans les stratégies compétitives de la Francie occidentale du $\mathrm{XI}^{\mathrm{e}}$ siècle}

Comment le couple intervient-il dans les stratégies compétitives, ou du moins quel témoignage en donnent les sources? Si les époux forment une nouvelle unité de vie, marquée au XI ${ }^{\mathrm{e}}$ siècle par l'association plus étroite de l'épouse, ils continuent néanmoins à appartenir à leur famille respective. Il s'agit donc d'analyser les relations non seulement au sein du couple, mais aussi entre celui-ci et les groupes familiaux dans lesquels il s'insère. Pour cela, trois cas de figure seront successivement envisagés: d'abord celui où le couple apparaît solidaire; ensuite, celui où les conjoints, tout en étant liés, se trouvent investis différemment dans la compétition; enfin, celui où ils sont en compétition l'un contre l'autre.

couple - compétition $-\mathrm{XI}^{\mathrm{e}}$ siècle - famille - élites

\section{Couple and Competitive Strategies in West Francia during the eleventh Century}

How does the couple intervene in competitive strategies or, at least, what testimony give documents about it? If the spouses form a new unit of life, marked in the eleventh century by the more narrow association of the wife, they continue nevertheless to belong to their respective family. We have to analyze therefore the relations not only within the couple, but also between the couple and the family groups he's part of. For this purpose, three cases will successively be considered: first, when the couple seems to be strongly united; second, when the spouses, even bounded, are invested differently in the competition; finally, when they are in competition the one against the other.

couple - competition - eleventh century - family - elites 